\title{
Knockout of Eva1a leads to rapid development of heart failure by impairing autophagy
}

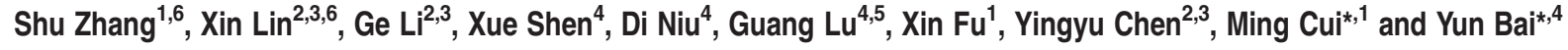

EVA1A (Eva-1 homologue $A$ ) is a novel lysosome and endoplasmic reticulum-associated protein that can regulate cell autophagy and apoptosis. Eva1a is expressed in the myocardium, but its function in myocytes has not yet been investigated. Therefore, we generated inducible, cardiomyocyte-specific Eva1a knockout mice with an aim to determine the role of Eva1a in cardiac remodelling in the adult heart. Data from experiments showed that loss of Eva1a in the adult heart increased cardiac fibrosis, promoted cardiac hypertrophy, and led to cardiomyopathy and death. Further investigation suggested that this effect was associated with impaired autophagy and increased apoptosis in Eva1a knockout hearts. Moreover, knockout of Eva1a activated Mtor signalling and the subsequent inhibition of autophagy. In addition, Eva1a knockout hearts showed disorganized sarcomere structure and mitochondrial misalignment and aggregation, leading to the lack of ATP generation. Collectively, these data demonstrated that Eva1a improves cardiac function and inhibits cardiac hypertrophy and fibrosis by increasing autophagy. In conclusion, our results demonstrated that Eva1a may have an important role in maintaining cardiac homeostasis.

Cell Death and Disease (2017) 8, e2586; doi:10.1038/cddis.2017.17; published online 2 February 2017

Cardiac remodelling is a pivotal pathological phenomenon that occurs during the clinical course of stress-induced heart failure and represents an independent risk factor for subsequent cardiac morbidity and mortality. ${ }^{1}$ Specifically, cardiac hypertrophy is characterized by an abnormal enlargement of the heart muscle as a result of the increased myocyte cell size and abnormal proliferation of non-muscle cells. ${ }^{2,3}$ Cardiac fibrosis is characterized by excessive extracellular matrix accumulation and fibroblast deposition, which eventually destroys organ architecture and abolishes normal function. ${ }^{4-6}$ Cardiac remodelling is a major biological determinant of fatal events, including heart failure, severe arrhythmias, and sudden cardiac death. ${ }^{7,8}$ Thus, elucidating the mechanisms implicated in cardiac protection against remodelling is of great significance.

Autophagy is a highly conserved catabolic process that is involved in delivering cytoplasmic components to lysosomes for degradation. It has a pivotal role in maintaining the cellular environment of the heart. ${ }^{9}$ Effective autophagy in cardiomyocytes is necessary for normal metabolism and cellular survival. The inability of autophagy to completely remove damaged structures results in a progressive accumulation of cellular debris, including cytosolic protein aggregates and defective mitochondria. Previous studies have demonstrated that dysregulation of autophagy can promote the development of many forms of heart disease as well as cardiac remodelling. ${ }^{10-14}$ Moreover, autophagy is inhibited during the progression of cardiac hypertrophy, which is an important part of cardiac remodelling. ${ }^{9}$ Studies have found that facilitation of autophagy can attenuate cardiac remodelling. ${ }^{15,16}$ However, the precise role of autophagy in cardiac remodelling remains to be elucidated.

The Eva-1 homologue A (EVA1A; also known as transmembrane protein 166 [TMEM166] and family with sequence similarity 176 [FAM176A]) is a novel lysosome and endoplasmic reticulum-associated protein that can regulate cell autophagy and apoptosis. ${ }^{17,18}$ It is conserved in humans, chimpanzees, rats, mice, and dogs, indicating that it may have important functions in vertebrates. Previous studies have shown that EVA1A is expressed in a cell-type- and tissue-typespecific manner, and is significantly downregulated in cancer tissues. ${ }^{18-20}$ Furthermore, in vivo and in vitro experiments have demonstrated that EVA1A overexpression inhibits tumour cell proliferation by both autophagy and apoptosis. ${ }^{19,20}$ Latest research shows that Eva1a deletion impairs the generation of newborn neurons by activating the PIK3CAAKT axis and inhibiting autophagy. ${ }^{21}$ However, its role in cardiac remodelling remains to be elucidated.

Therefore, we aimed to determine the role of Eva 1a in cardiac remodelling in the adult heart. To this end, we generated an inducible myocyte-specific Eva1a knockout mouse model to investigate the role of Eva1a in the adult heart.

\section{Results}

Generation of tissue-specific Eva1a knockout mice. Consistent with previous reports, RT-PCR results showed that Eva1a mRNA was moderately expressed in adult heart

\footnotetext{
${ }^{1}$ Department of Cardiology, Peking University Third Hospital, Beijing 100191, China; ${ }^{2}$ Department of Immunology, School of Basic Medical Sciences, Peking University Health Science Center, Beijing 100191, China; ${ }^{3}$ Peking University Center for Human Disease Genomics, Beijing 100191, China; ${ }^{4}$ Department of Cell Biology, School of Basic Medical Sciences, Peking University Health Science Center, Beijing 100191, China and ${ }^{5}$ Department of Physiology, Yong Loo Lin School of Medicine, National University of Singapore, Singapore 117597

${ }^{*}$ Corresponding author: M Cui or Y Bai, Department of Cell Biology, Peking University Health Science Center, Haidian District, Xueyuan Road 38, Beijing 100191, China. Tel: +86 10 82801495. E-mail: mingcui@ bjmu.edu.cn or baiyun@bjmu.edu.cn

${ }^{6}$ These authors contributed equally to this work and share the first authorship.

Received 03.9.16; revised 09.1.17; accepted 09.1.17; Edited by GM Fimia
} 


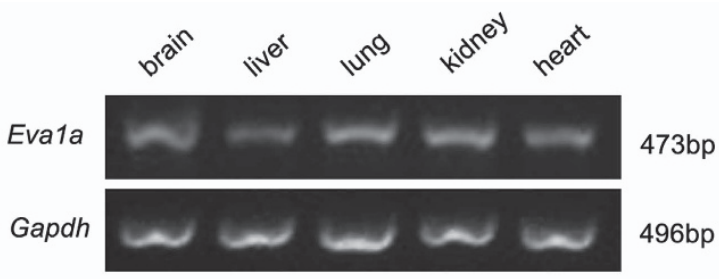

C

Wild-type Eva1a gene
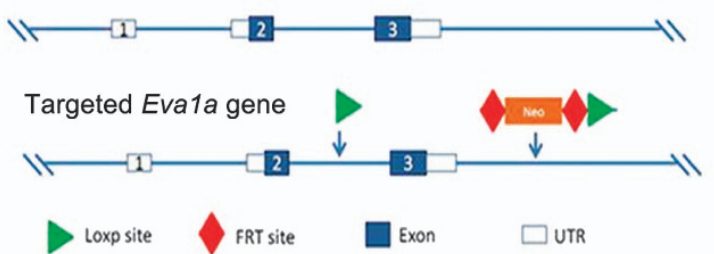

b

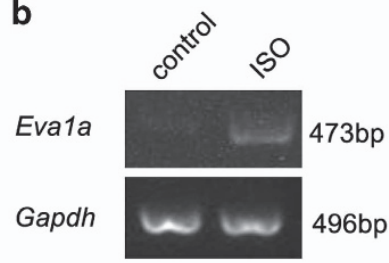

d

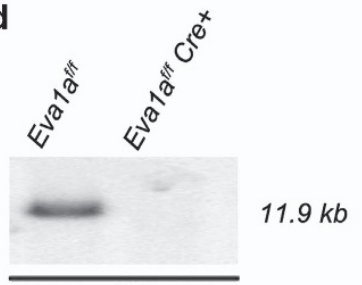

tamoxifen e

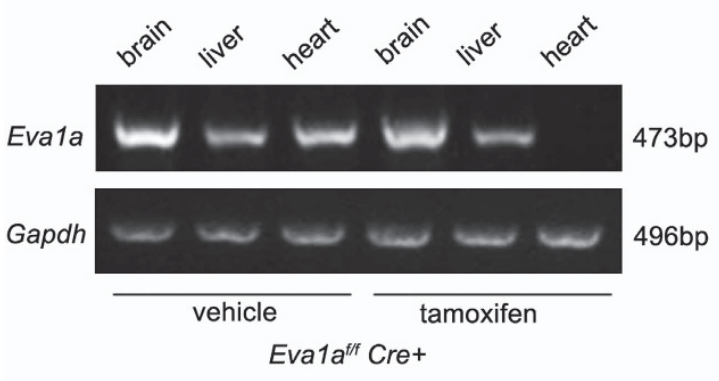

f

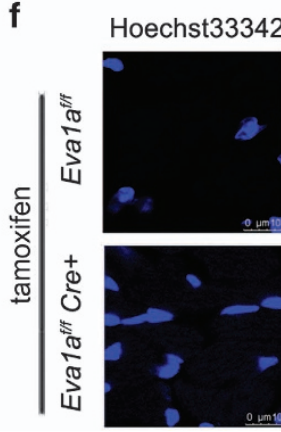

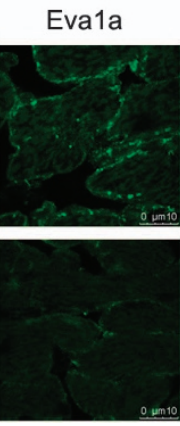

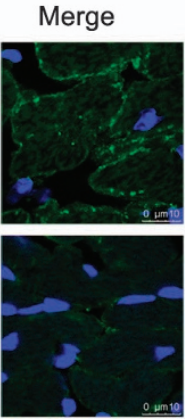

Figure 1 Characterization of Eva1a expression in Eva1 ${ }^{f / f}$ and Eva1a-deficient mice. (a) Representative RT-PCR results showing endogenous Eva1a expression in different tissues from wild-type mice. (b) Representative RT-PCR results showing endogenous Eva1a expression in ISO-induced cardiac remodelling. (c) Scheme to generate Eva1adeficient mice. (d) Southern blot analysis to detect Eva1a expression in the heart in Eva1a ${ }^{\text {t/f }}$ and Eva1a-deficient mice. (e) Representative RT-PCR results showing endogenous Eva1a expression in different tissues from Eva1a ${ }^{\text {f/t }}$ and Eva1a-deficient mice. (f) Eva1a expression was detected by immunofluorescence assay

tissue (Figure 1a). To determine whether Eva1a is involved in cardiac remodelling, we examined the gene expression in heart extracts from adult mice after they were treated with isoproterenol (ISO) for 2 weeks. As shown in Figure 1b, there was a significant increase in Eva1a expression in ISOinduced hypertrophied hearts, indicating its potential involvement in cardiac remodelling. To determine the role of Eva1a in cardiac remodelling, we generated temporally controlled cardiac-specific Eva1a-deficient mice. Eva1a flox/flox mice had two LoxP sequences flanking exon 3 of the mouse Eva1a gene and a neo cassette. Cre-mediated deletion led to a deletion mutation because of direct splicing from exon 3 and the neo cassette, producing a small truncated non-functional peptide (Figure 1c). Southern blot analysis was used to confirm the deletion of Eva1a in heart tissue by a tamoxifeninducible Cre recombinase (MerCreMer, Figure 1d). The resulting Eva1a flox/flox $:$ a $M H C$ MerCreMer ${ }^{+}\left(\mathrm{Eva1a}^{\mathrm{f} / f} \mathrm{Cre}^{+}\right)$ mice were indistinguishable in appearance from their agematched control Eva1a flox/flox $: a-M H C$ MerCreMer ${ }^{-}\left(\mathrm{Eva1a}^{\mathrm{f} / \mathrm{f}}\right)$ littermates. In the $\mathrm{Eva}^{\mathrm{f}} \mathrm{a}^{\mathrm{f} f} \mathrm{Cre} \mathrm{Cr}^{+}$mice that had been treated with tamoxifen for 3 days, Eva1a-deficient mice were identified by RT-PCR and immunofluorescence analyses, and Eva1a gene and protein expression levels were significantly reduced in the heart of $\mathrm{Eva}^{\mathrm{ff/f}} \mathrm{Cre}^{+}$mice (Figures 1e and f).
Loss of Eva1a leads to rapid early mortality and contractile dysfunction. Comparison of the survival rates between Eva1a-ko mice and their littermates with the Eva1a ${ }^{f / f}$ genotype revealed a significant difference between these two groups. Eva1a-ko mice that were treated with tamoxifen for 3 days started to die, and fewer than half of the mice survived to 28 days (Figure 2a).

Echocardiography was performed to determine alterations in the cardiac structure and function of Eva1a-ko mice. The left ventricular end-diastolic diameter (LVID-d) and left ventricular end-systolic diameter (LVID-s) were both higher in the Eva1a knockout mice than in the other groups (Figures $2 \mathrm{~b}$ and $\mathrm{d}$ ). Left ventricular function was severely impaired in Eva1a knockout mice as indicated by a significant decline in percent fractional shortening (FS) and a significant decrease in the ejection fraction (EF; Figures $2 b$ and $c$ ), whereas the FS and EF values in the other groups showed no marked differences. In addition, the diastolic left ventricular wall thickness (LVPW-d) did not significantly differ between Eva1a knockout mice and the other groups (Figures $2 \mathrm{~b}$ and e). Consistent with these findings, dissection of anaesthetised moribund Eva1a-deficient mice showed obvious pulmonary oedema, and the lung weight normalized to the body weight was significantly higher in the Eva1a-deficient mice than in the other groups (Figure $3 \mathrm{c}$ ). 


\section{a}

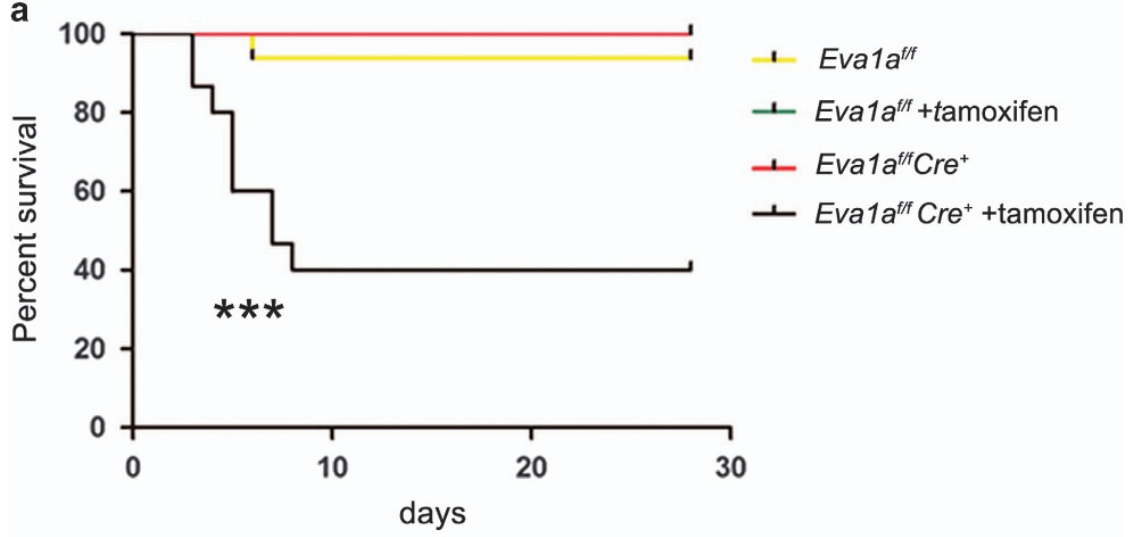

b

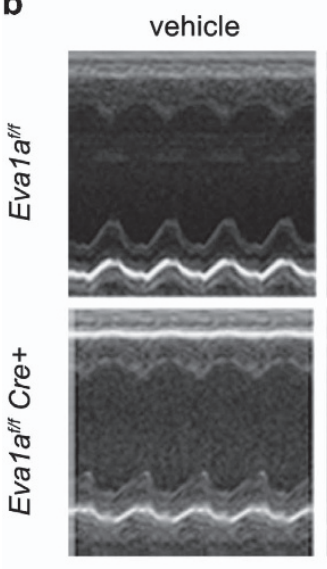

d

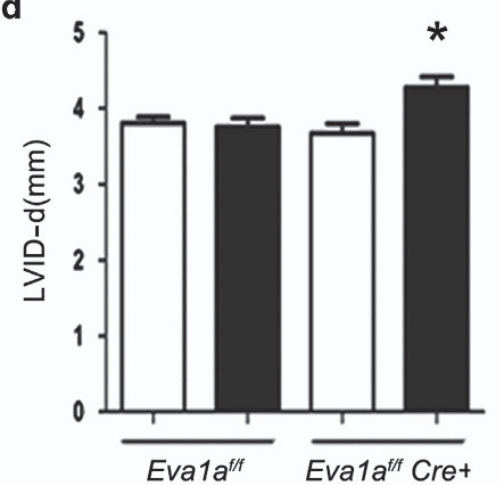

C
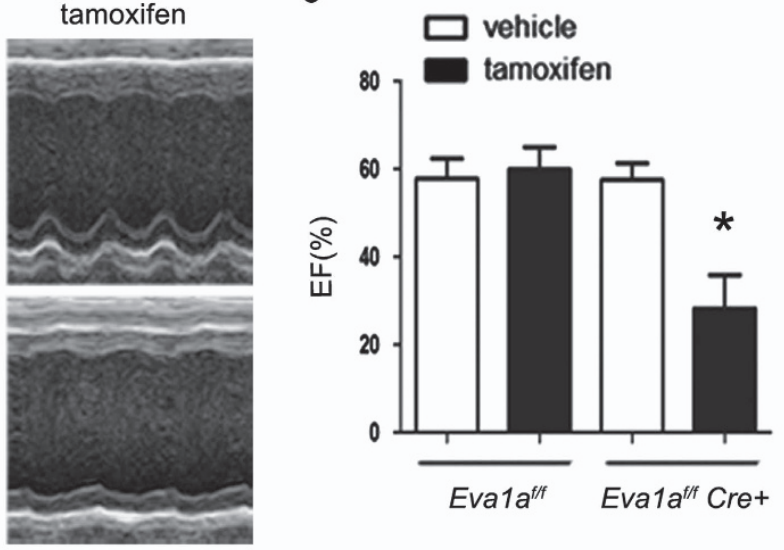

e

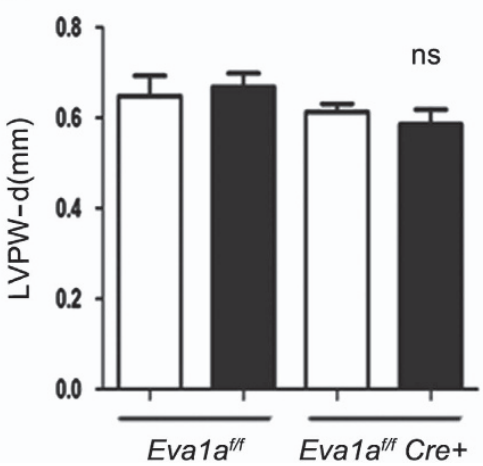

Figure 2 Effects of Eva1a-deficiency on cardiac function and structure. (a) Kaplan-Meyer survival curve (** $P<0.0001, n=10)$. (b) Representative M-mode echocardiography images showing left ventricular wall thickness and systolic function in different mice. (c) Ejection fraction (EF) was significantly lower in Eva1a-deficient mice than in the other mice $\left({ }^{*} P<0.05, n=8\right)$. (d) Echocardiographic analysis revealed enlarged left ventricular diastolic dimension (LVID-d) was significantly higher in Eva1a-deficient mice than in the other mice $\left({ }^{*} P<0.05, n=8\right)$. (e) Diastolic left ventricular wall thickness (LVPW-d) did not significantly differ between Eva1a-deficient mice and the other groups $(n=8)$

The mice were analysed for several cardiac remodellingrelated indicators at 1 day before tamoxifen injection and at 3,7 , and 14 days post tamoxifen injection. At 3 days post tamoxifen injection, the LVID-d was greater in the Eva1adeficient group than in the other groups; there was also a marked difference at 14 days (Figure $2 d$ ). In addition, the EF and FS values were significantly lower in the Eva1a-deficient group than in the other groups from 3 days after the injection (Figure 2c). Collectively, these results indicate that Eva1adeficient mice displayed worse protection of cardiac function than the other mice.
Knockout of Eva1a promotes cardiac hypertrophy.

Cardiac hypertrophy is a major predictor for the development of cardiac remodelling. We carried out pathological analysis to investigate the role of Eva1a in cardiac hypertrophy. As shown in Figures $3 a$ and $b$, the heart weight/body weight (HW/BW) ratio was dramatically higher in the Eva1a-deficient mice than in the other groups (Figures $3 a$ and b). In addition, the cross-sectional cardiomyocyte area was higher in the Eva1a-deficient mice than in the other groups. However, there was no significant difference between the groups expressing Eva1a (Figures $3 a$ and d). The expression of 
a
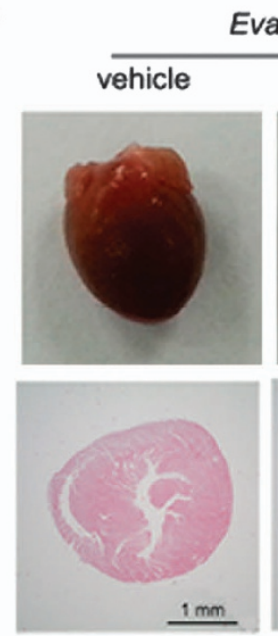

$1 \mathrm{~mm}$

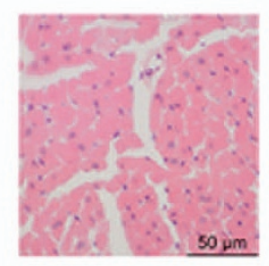

Eva1att tamoxifen
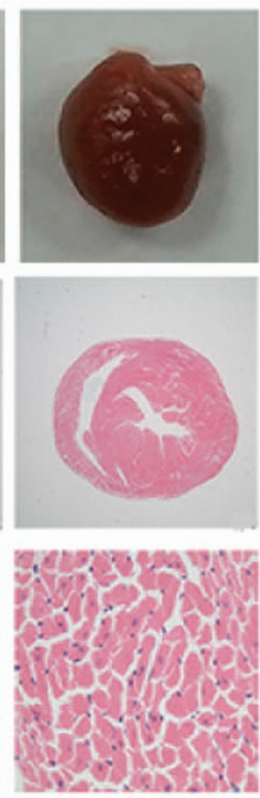

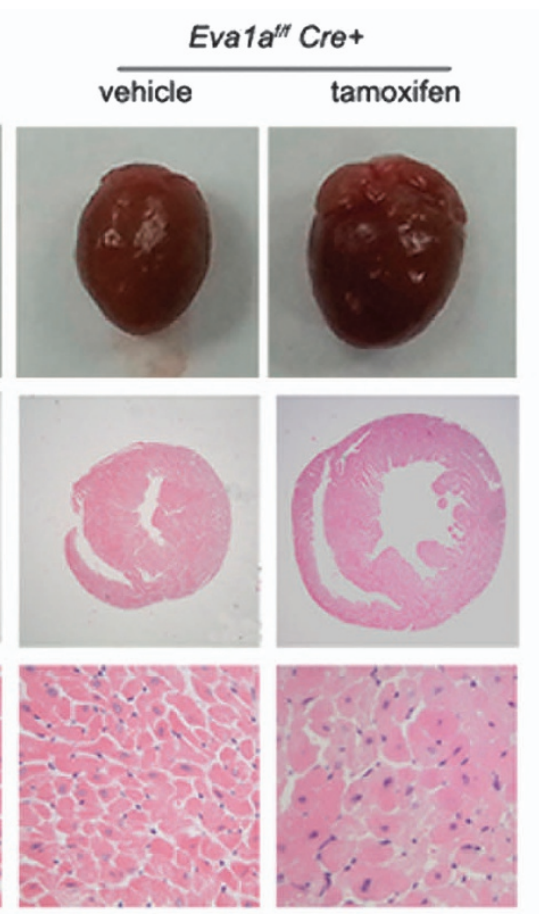

e

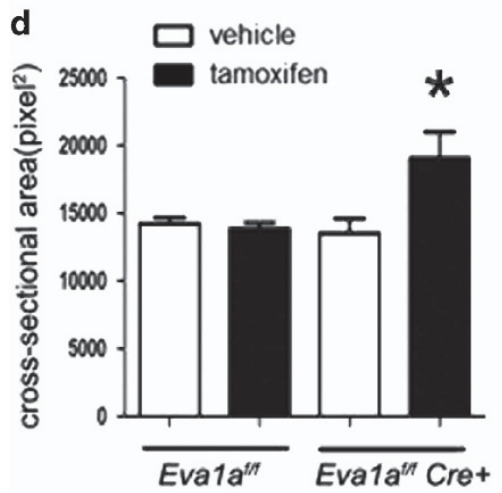

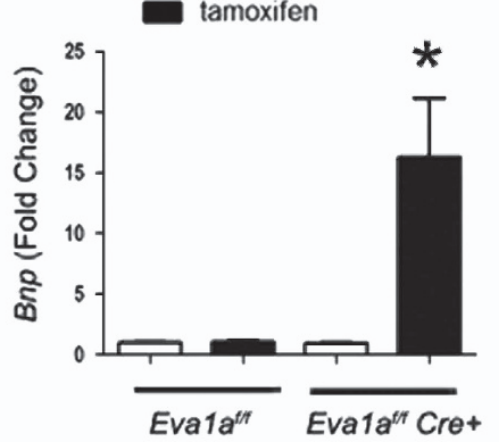
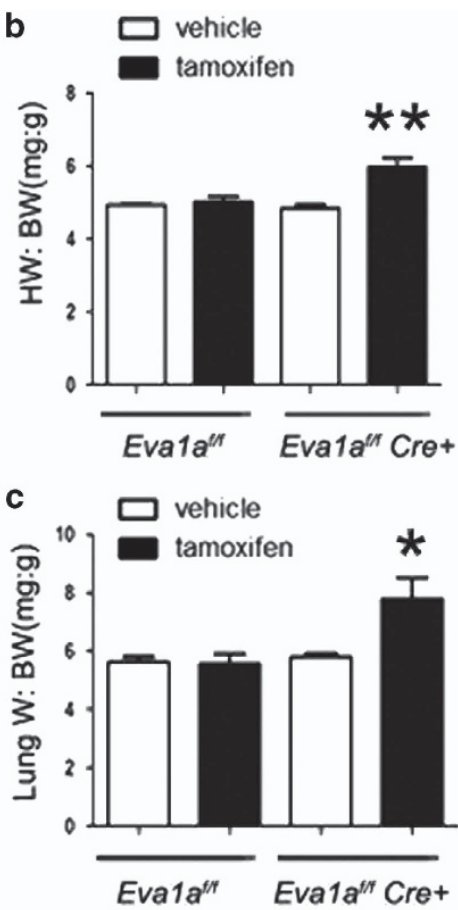

f
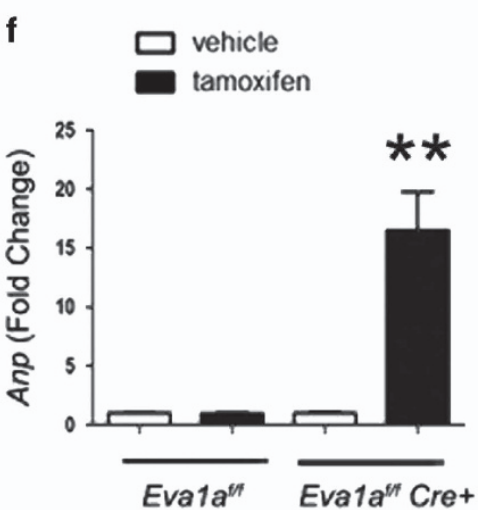

Figure 3 Effects of Eva1a deficiency on cardiac hypertrophy. (a) The hearts of Eva1a deficient mice were enlarged on gross morphology. Haematoxylin and eosin (H\&E) staining of heart sections. Scale bar $=1 \mathrm{~mm}$ or $50 \mu \mathrm{m}$. (b) The ratio of heart weight to body weight (HW/BW) did not significantly differ between Eva1a-deficient mice and the other mice $\left({ }^{* *} P<0.01, n=9\right.$ ). (c) The ratio of lung weight to body weight (LW/BW) was significantly different between Eva1a-deficient mice and the other mice $\left({ }^{*} P<0.05, n=9\right)$. (d) Measurements of two-dimensional cardiomyocyte cross-sectional areas $\left({ }^{*} P<0.05, n=4\right)$. (e and f) Analysis of hypertrophy markers Bnp (e) and Anp (f) by qRT-PCR $\left({ }^{*} P<0.05,{ }^{* *} P<0.01, n=5\right)$

the hypertrophic markers Anp and Bnp were significantly increased in the Eva1a knockout group, but the $\beta-M h c$ expression was not increased (Figures $3 e$ and f). Taken together, these results indicated that Eva1a may exert a beneficial effect on cardiac hypertrophy.

Eva1a knockout increases cardiac fibrosis in vivo. Cardiac fibrosis is an important process in pathological cardiac remodelling and can lead to heart failure. Therefore, we investigated the effect of Eva1a on cardiac fibrosis by Sirius red staining. At 3 days post tamoxifen injection, collagen accumulation was absent in all the groups (data not shown); however, collagen deposition was increased in the Eva1a-ko heart tissues at 2 weeks after injection (Figure 4a), whereas collagen accumulation was absent in the other groups (Figure $4 a$ ). Results of the quantitative analysis revealed that the collagen volume in the myocardium was lower in the other groups than in the Eva1a-deficient group (Figure 4b). There was decreased fibrosis in the groups with Eva1a; this may be because of the increased collagen degradation or decreased collagen synthesis in response to tissue damage. Fibrosis is characterized by excessive accumulation of collagen and other extracellular matrix components. Development of the fibrotic extracellular matrix is driven by connective tissue growth factor (CTGF) and other cytokines and growth factors, with overload of factors such as collagen I, collagen III, fibronectin. ${ }^{22}$ Quantitative RT-PCR was performed and the levels of Ctgf, Collagen I, Collagen III, and Fibronectin were found to be increased in the Eva1a-deficient group (Figures 4c-f). Thus, the results of these experiments suggest that Eva1a expression in mice attenuates cardiac fibrosis. 
a
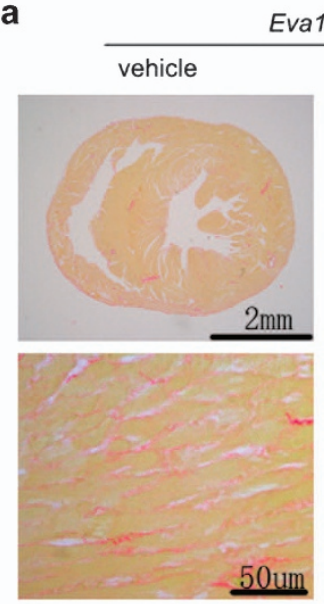

$\underline{50 u m}$

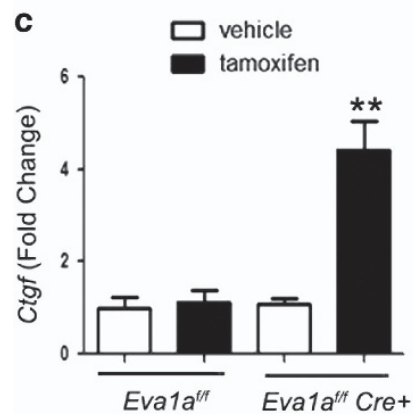

va1 $a^{f f t}$
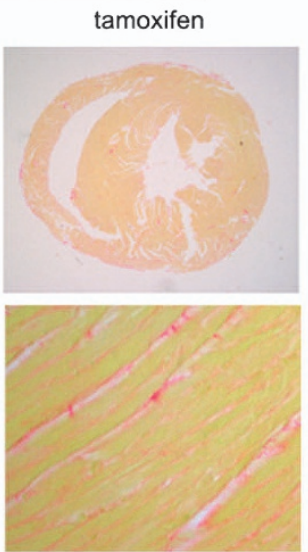
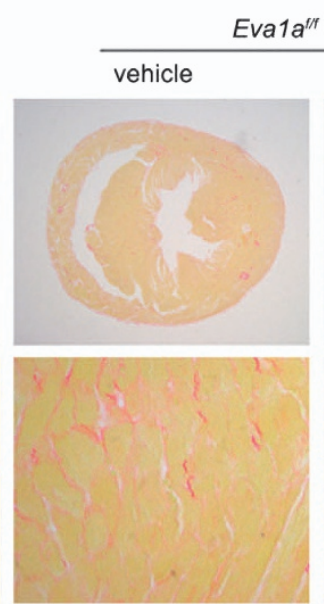

va1 $a^{\prime \prime}$ Cre+

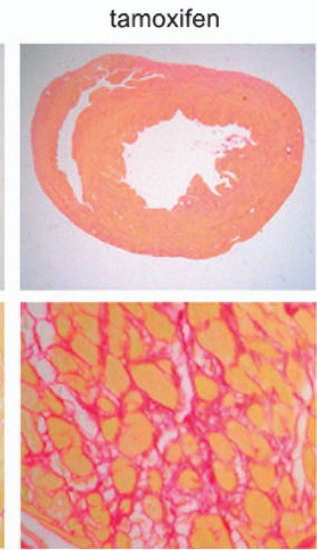

b
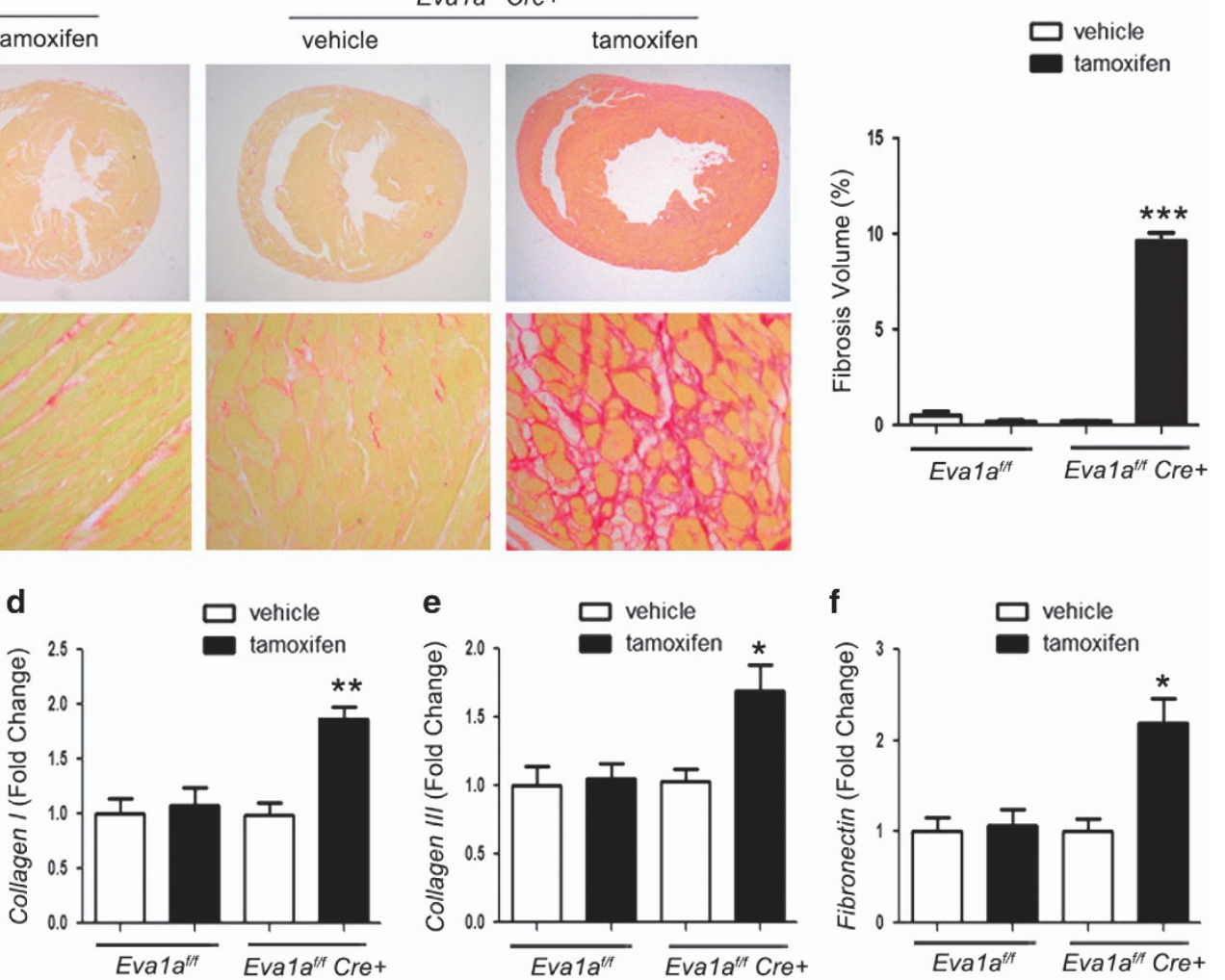

Figure 4 Effects of Eva1a deficiency on cardiac fibrosis. (a) Representative micrographs of picrosirius red-stained sections of the ventricle. Red areas represent collagen. Scale bar $=2 \mathrm{~mm}$ or $50 \mu \mathrm{m}$. (b) Quantification of cardiac interstitial collagen content in picrosirius red-stained sections. Results are expressed as the ratio of collagen area to heart area $\left({ }^{* \star *} P<0.001, n=4\right)$. (c-f) Analysis of fibrosis markers Ctgf $(\mathbf{c})$, Collagen I (d), Collagen III (e), and Fibronectin (f) by qRT-PCR $\left({ }^{*} P<0.05,{ }^{* *} P<0.01, n=6\right)$

Loss of Eva1a decreases autophagy and increases apoptosis in heart tissue. Previous studies have shown that autophagy increases protein turnover during cardiac remodelling. ${ }^{23}$ Western blot analysis was carried out to study the expression levels of two autophagy-associated markers, Lc3b and Sqstm1. The results of the western blot analysis showed that the Lc3b-II/Gapdh level was significantly lower in the Eva1a-deficiency group than in the other groups (Figures 5a and c), whereas the Lc3b-II/Gapdh level in the other groups were not significantly different (Figures $5 \mathrm{a}$ and c). It has been widely recognized that the Sqstm1 protein serves as a link between Lc3 and ubiquitinated substrates. It incorporates into the completed autophagosome and is degraded in lysosomes. Therefore, the Sqstm1 level can serve as a marker for autophagic flux. Our results showed that Sqstm 1 expression was lower at the baseline than after tamoxifen treatment, and there were significant differences in the expression levels between the vehicle- and tamoxifentreated groups (Figures $5 \mathrm{a}$ and $\mathrm{b}$ ). Consistent with this result, more ubiquitinated inclusions accumulated in the heart tissue in Eva1a-deficiency group (Supplementary Figures S1a). Furthermore, Nbr1, another well-characterized autophagy substrate, also dramatically elevated in the Eva1a-deleted heart tissue, but not in the wild-type heart tissue (Supplementary Figures S4). These results indicate that loss of Eva1a leads to a significant decrease of autophagic flux.
As apoptosis and autophagy have simultaneously been observed in the failing human heart and as EVA1A is known for its important role in apoptosis regulation, ${ }^{18,20}$ we asked whether cardiac remodelling is associated with apoptosis. To answer this question, we performed a terminal deoxynucleotidyl transferase-mediated dUDP nick-end labelling (TUNEL) assay using heart sections from all four groups. Results of the TUNEL assay revealed apoptotic cells in all the mice groups. The Eva1a-deficient mice had a significantly higher proportion of apoptotic cells than other mice (Figures $5 d$ and e). We also examined cleaved caspase-3, an activated form of caspase- 3 that acts as a lethal protease at the most distal stage of the apoptosis pathway. Our results revealed that the cleaved caspase-3 signalling was significantly higher in Eva1a-deficient mice than in other mice (Supplementary Figures S1b). However, there was no significant difference in the proportion of apoptotic cells between the vehicle-treated groups (data not shown). These data suggest that apoptosis may have exacerbated cardiac remodelling in Eva1adeficient mice.

Loss of Eva1a in heart affects energy metabolism. Transmission electron microscopy (TEM) of Eva1a-deficient hearts showed disorganized sarcomere structure, mitochondrial misalignment, and aggregation (Figure 5f). As the heart is rich in mitochondria, which is closely related to energy 
a

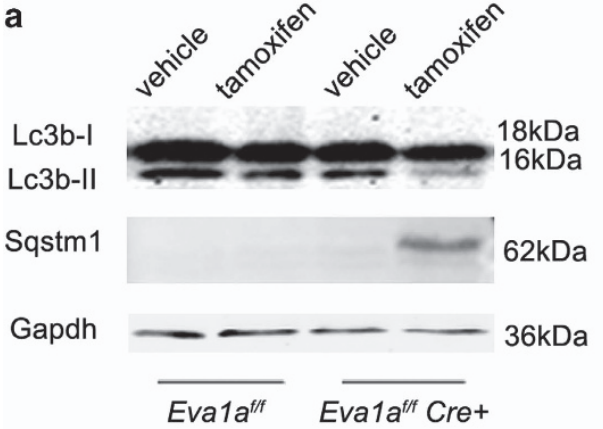

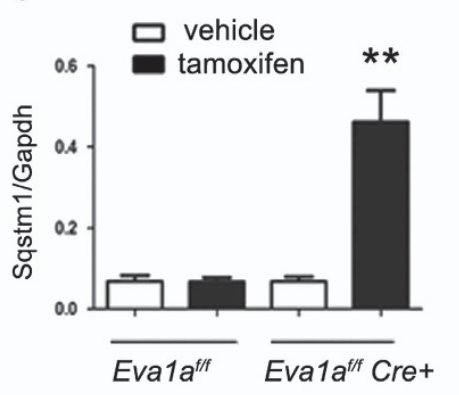

C

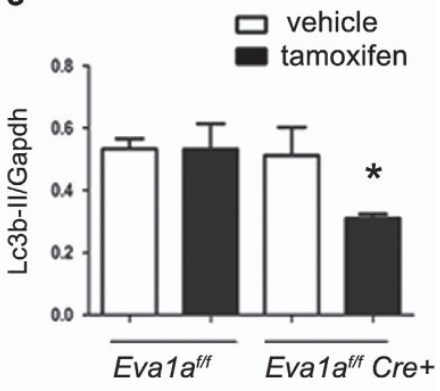

d

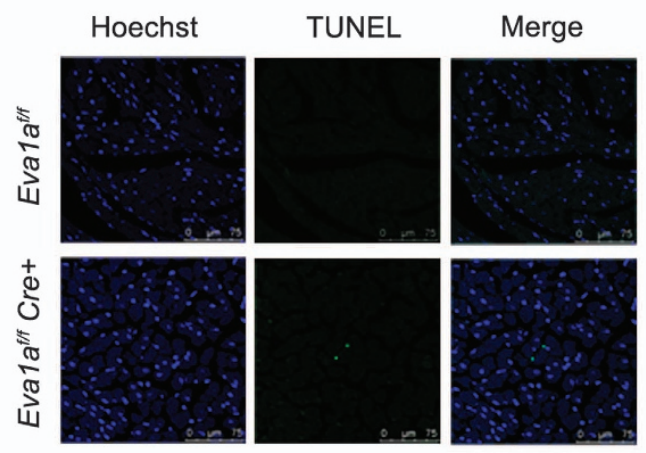

f

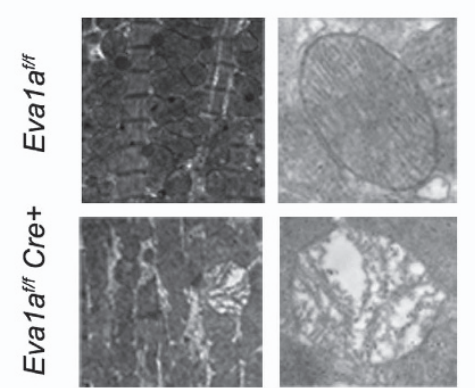

e

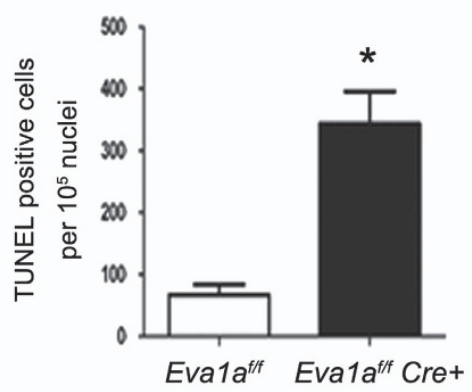

g

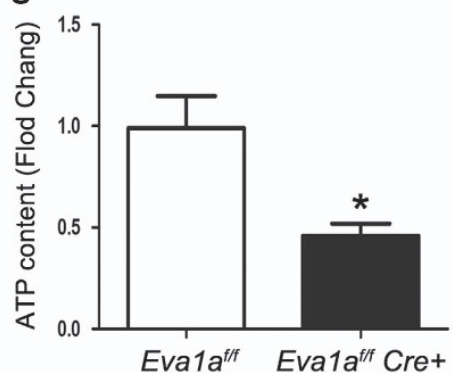

Figure 5 Assessment of autophagy and apoptosis in Eva1a-deficient mice. (a) Representative western blot analysis of Lc3b and Sqstm1 in heart extracts obtained from different groups. (b and $\mathbf{c}$ ) Densitometric analysis of Sqstm1 and Lc3b-II ( $\left.{ }^{*} P<0.05,{ }^{* *} P<0.01, n=4\right)$. (d) Representative images of TUNEL staining (green) and Hoechst staining (blue) of nuclei in cryosectioned heart tissue. (e) Quantification of positive cells displaying terminal deoxynucleotidyl transferase-mediated dUDP nick-end labelling (TUNEL) staining. (f) Ultrastructural images reveal the presence of swollen mitochondria and lipid accumulation in Eva1a-deficient mice. (g) Detection of ATP levels $\left({ }^{*} P<0.05\right.$, $n=3)$

metabolism, we investigated whether deficiency of Eva1a led to the lack of ATP generation. An ATP kit was used to detect the ATP content in the heart, and the results revealed a significantly lower ATP content in Eva1a-deficient mice than in other mice (Figure $5 \mathrm{~g}$ ), suggesting that Eva1a may have a role in mitochondrial energy metabolism and regulation of cardiac remodelling.

Owing to the disorganized mitochondria observed in Eva1a-deficient mice hearts, we studied some of the mitochondria-related proteins. The levels of Drp1, which mediates mitochondrial division, ${ }^{24}$ were also downregulated in Eva1a-deficient mice (Supplementary Figures S2a and b). Tomm20, which is a mitochondrial marker, ${ }^{25}$ was accumulated in the Eva1a knockout group (Supplementary Figures S2a and c). The other proteins detected, including Pink1, Parkin, Bnip3, mitofusin2, and Pgc1, revealed no obvious changes (Supplementary Figures S2a). Taken together, our results suggested that loss of Eva1a led to the inhibition of autophagy, resulting in the accumulation of some impaired mitochondria in the hearts of Eva1a knockout mice.

Eva1a modulates autophagy through the LKB1/AMPKMtor pathway. We then investigated the mechanisms of the defective autophagy in Eva1a-deletion mice. The mammalian target of rapamycin (Mtor) has been known as a negative regulator of autophagy. The results showed that the phosphorylation levels of Mtor at Ser2448 (activation site) and Rps6kb1 (70-kDa ribosomal protein S6 kinase, polypeptide 1), as well as Eif4ebp1 (eukaryotic translation initiation factor 4E binding protein 1), two downstream effectors of Mtor were elevated in Eva1a knockout hearts (Figures 6a-c, Supplementary Figure S3a and b). In addition, the levels of total and phosphorylated Ulk1 at Ser555 (activation site) ${ }^{26}$ were both decreased in Eva1a-deficient mouse hearts 

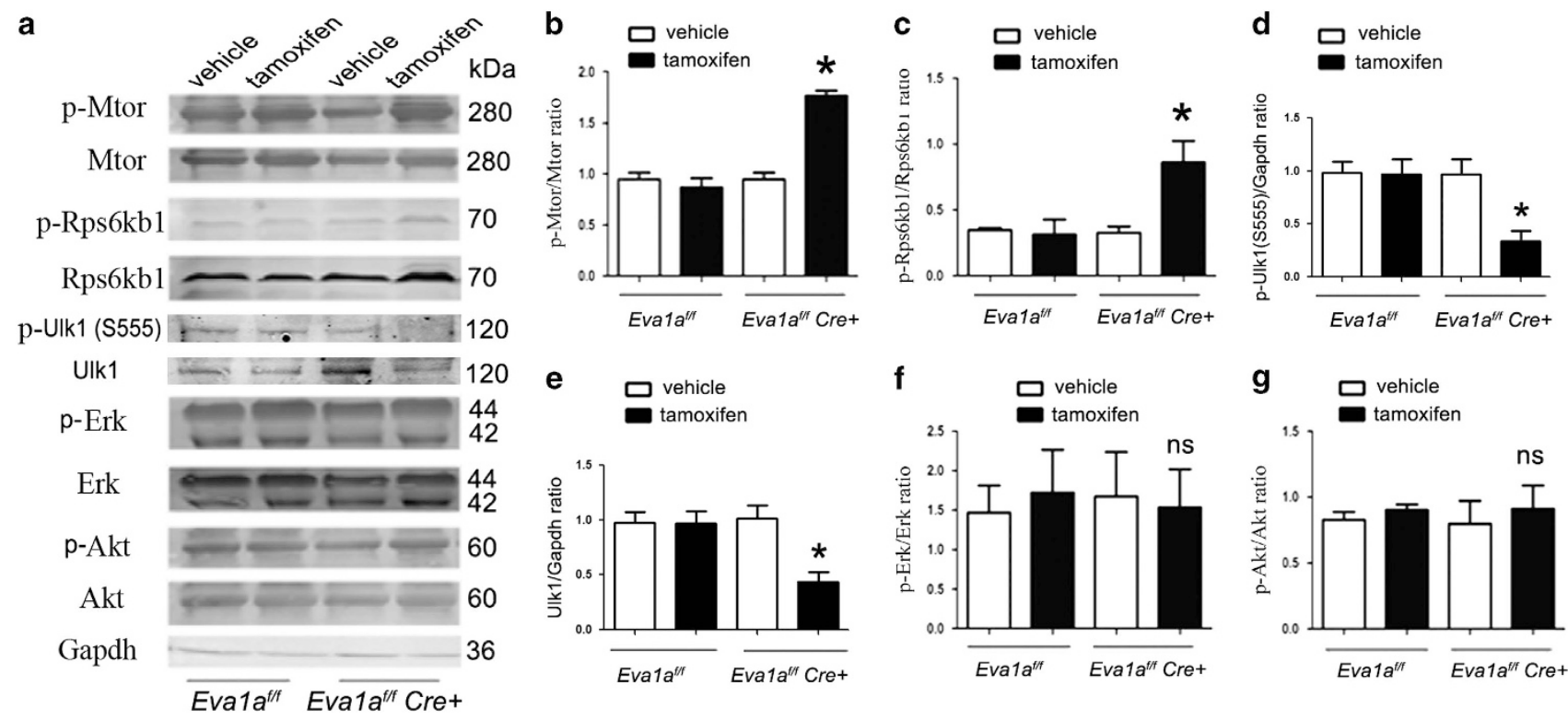

Figure 6 Eva1a deficiency increases the Mtor signalling pathway. (a) Representative western blot analysis of Mtor, Rps6kb1, Ulk1, Erk, and Akt, and their phosphorylated forms in heart extracts obtained from different groups of mice. $(\mathbf{b}-\mathbf{g})$ Densitometric analysis of $p$-Mtor, $p$-Rps6kb1, $p$-Erk, and $p$-Akt $\left({ }^{*} P<0.05, n=3\right)$

(Figures 6a, d, and e). These data implied that the defective autophagy in Eva1a knockout mice might be related to Mtor activation and Ulk1 downregulation.

The main regulatory molecules upstream of the Mtor signalling pathway include PIK3CA/AKT, MAPK/ERK, and LKB1/AMPK. ${ }^{18,27-29}$ Therefore, we evaluated the phosphorylation levels of Akt, Erk, Lkb1, and Ampk, and the results revealed that the phosphorylation levels of Akt and Erk in heart tissue did not differ between the Eva1a knockout mice and other mice (Figures 6a, f, and g), but Eva1a deletion reduced the phosphorylation levels of Lkb1 and Ampk (Supplementary Figures S3c-e).

To investigate the significant involvement of Mtor and autophagy in the development of the cardiac phenotype, we used the Mtor-specific inhibitor rapamycin for the rescue experiments. The results indicate that rapamycin could partially rescue left ventricular function by increasing the EF in the Eva1a-deficiency group, and reduces the level of the hypertrophic markers Anp and Bnp, suggesting that the cardiac dysfunction induced by Eva1a $\mathrm{KO}$ is mediated by Mtor activation (data not shown). Taken together, these results suggested that loss of Eva1a leads to the inhibition of the Lkb1-Ampk axis, resulting in the activation of Mtor signalling and decreased autophagy in the Eva1a knockout hearts.

\section{Discussion}

In the present study, we investigated the role of Eva1a in cardiac remodelling using an Eva1a knockout mice model. We demonstrated that Eva1a attenuated cardiac remodelling by modulating autophagy. Genetic disruption of Eva1a disrupted the autophagic machinery, consequently impairing cardiac homeostasis, leading to mitochondrial damage, and decreasing the ATP content, and the clearance of impaired mitochondria. This effect may be associated with the decreased autophagy via the Lkb1/Ampk-Mtor signalling pathway. The results of our study suggested that Eva1a may be a promising therapeutic candidate for heart disease.

Heart failure has become one of the most serious threats to human health worldwide. Cardiac remodelling, which is triggered by a variety of factors, is a factor initiating heart failure. Therefore, exploring the molecular mechanisms underlying cardiac remodelling can shed insights into the clinical prevention and treatment of heart failure. Autophagy is a complex metabolic programme that is closely related to many diseases, and it has an important role in the regulation of cardiovascular system.

Eva1a is a novel membrane protein that is an important autophagy- and apoptosis-related molecule. Several previous studies have demonstrated the association of this protein with many pathological conditions and diseases. ${ }^{18-20,30}$ However, the role of Eva1a in cardiac remodelling remains unknown. Our results demonstrated moderate Eva1a mRNA expression in the adult heart tissue. In addition, we first examined gene expression in heart extracts from adult mice subjected to ISO treatment for 2 weeks. There was a significant increase in the Eva1a expression in ISO-induced hypertrophied hearts. These data suggest that Eva1a is potentially involved in cardiac remodelling.

In this study, we examined the cardiac structure and function of mice using small-animal ultrasound equipment. Our results indicated that the EF and FS were significantly lower in the Eva1a-deficient mice on the third day after injection of tamoxifen and the LVID-d was significantly increased; however, the LVPW-d showed no marked difference between the groups. The results of the above suggest that the Eva1adeficient mice showed a reduction in cardiac function and cardiac centrifugal expansion. These observations are supported by similar findings in Atg5- and MCL-1-deficient genetic models. ${ }^{15,30,31}$ We developed an Eva1a knockout mice model, 
and Eva1a was systemically knocked out in Eva1a ${ }^{-/}$mice during embryonic development. We found no significant alterations in the birth and survival rate, and no obvious abnormalities in cardiac function in adult mice (data not shown). We hypothesized that the different phenotypes of systemic Eva1a knockout mice and adult inducible cardiacspecific Eva1a-deficient mice may be due to the embryonic stage of development-related genes compensation, which is similar to the results reported in some international studies. ${ }^{9,15}$ Moreover, no international studies have investigated the role of Eva1a in cardiac function, and our study demonstrates that Eva1a has a protective effect on cardiac function at baseline.

Pathological changes are of unique significance in the diagnosis of myocardial hypertrophy. Histomorphological characteristics can reflect the extent of myocardial damage; furthermore, they can be studied to determine the curative effect of therapy. Our study showed that the heart weight/body weight of Eva1a-deficient mice showed a significant increase compared with the other groups. Haematoxylin and eosin (H\&E) staining of Eva1a-deficient heart tissue revealed disorganized cardiomyocytes, cardiac hypertrophy, and swollen cytoplasm. The cross-sectional area of cardiomyocytes was significantly higher in Eva1a-deficient mice compared with that in the other mice groups. Currently, the embryonic genes Anp and Bnp have been used as important cardiac hypertrophy markers. Compared with other groups, Anp and Bnp were markedly increased in Eva1a-deficient mice; however, there was no significant difference in the $\beta$-Mhc expression between the four groups. This is the first study to discover that Eva1a deficiency can cause myocardial hypertrophy, indicating the important role that Eva1a had in maintaining cardiac homeostasis.

Structural changes in cardiac cells are another important manifestation of cardiac fibrosis, and it occurs as proliferation of a large number of fibroblasts and deposition of collagen fibre. Sirius red staining may reflect the interstitial remodelling in the myocardium, which mainly occurs for the reconstruction of the collagen fibre network; therefore, it is often used to detect the degree of cardiac fibrosis. In our study, none of the mice exhibited myocardial fibrosis on the third day (data not shown); however, partial Eva1a-deficient mice exhibited obvious cardiac fibrosis at 2 weeks. This may be due to the gradual development of fibrosis. On day 3 after tamoxifen induction, cardiac fibrosis in Eva1a-deficient mice may be too acute for compensatory mechanisms to be effective. Meanwhile, the results of the ultrasound analysis indicated that the cardiac function of Eva1a-deficient mice had improved at 2 weeks compared with that on day 3 . We consider that myocardial fibrosis may be caused by a compensatory response in the heart. On the other hand, oxidative and subsequent nitrosative damage of the myocardium and vasculature have been described as major primary mechanisms leading to pathological alterations associated with many cardiovascular diseases. Autophagy and oxidative stress are closely related in cardiomyopathy; the fibrosis also may be due to increased oxidative stress. ${ }^{32}$ A more detailed mechanism is still in progress.

Cardiomyocytes are widely used in the research of cardiac autophagy at the baseline. Autophagy can be a protective mechanism in cardiomyocytes under normal or mild levels of stress. As damage to the mitochondria could release proapoptotic factors, autophagy can prevent apoptosis activity and consequently inhibit myocardial apoptosis. Thus, it is believed that the protective effect of autophagy is related to its inhibition of apoptosis; and by providing amino acids, fatty acids, and other substances. Moreover, autophagy has a role in maintaining the cytoplasm and quantity, and degrades damaged organelles and proteins to provide ATP.

Previous studies have shown that autophagy increases protein turnover during cardiac remodelling. ${ }^{23}$ Therefore, we examined the expression of two autophagy markers, Lc3b and Sqstm1. The Lc3b-II/Gapdh level was significantly decreased and the Sqstm1 expression level was significantly increased in Eva1a-deficient mice compared with the other mice, indicating that the autophagy in myocardial tissue was decreased in Eva1a-deficient mice. Collectively, these data suggest that Eva1a may have a beneficial role in the heart via autophagy. Abnormal autophagy could lead to cell death. ${ }^{9,30}$

The results of our study revealed a significantly higher proportion of apoptotic cells in the myocardial tissue of Eva1a-deficient mice than in the other groups, indicating that abnormal autophagy that occurs after the loss of Eva1a, which eventually leads to increased apoptosis, may be one of the reasons for the cardiac remodelling.

The autophagy-initiating factor ULK1, which belongs to the serine/threonine kinase family, is required for autophagy induction. ${ }^{27,33}$ ULK1 forms a complex with Atg13 and FIP200 to regulate the initial step of autophagy induction in mammalian cells. In addition, Ampk-dependent phosphorylation of Ulk1 (S555) is critical for translocation of Ulk1 to mitochondria and promotion of mitophagy. Considering the disorganized mitochondria in Eva1a-deficient mice hearts, the levels of total Ulk1 and phosphorylated Ulk1 at Ser555 (activation site) are both decreased in Eva1a-deficient mouse hearts (Figures 6a, e and f). Drp1 is located in the mitochondrial outer membrane and mediates mitochondrial division. In Drp1-deleted cardiomyocytes, mitochondria exhibited increased connectivity, accumulated ubiquitinated proteins, and decreased respiration, ${ }^{25}$ which is similar to that observed in Eva1a-deleted mice. This suggests that Eva1a deletion may inhibit mitophagy by downregulating Drp1 expression in some degree. The inhibition of mitophagy lead to damaged mitochondria that cannot be degraded and accumulation of the mitochondrial marker Tomm20.

There is a close relationship between autophagy and energy metabolism. In the normal heart, mitochondrial autophagy occurs to maintain mitochondrial function, promote the generation of mitochondria, and to increase the generation of energy. In addition, autophagy degrades long-lived proteins, producing amino acids that generate ATP in the tricarboxylic acid cycle. We wondered whether the decreased autophagy in Eva1a knockout hearts could affect the normal function of mitochondria, resulting in energy metabolism disorders, and consequently influence cardiac remodelling. The results of the electron microscopic analysis demonstrated morphological abnormalities, disordered arrangement, and swelling in the mitochondria of Eva1a-deficient hearts; moreover, the mitochondria appeared blurred due to lack of particles in the electron-lucent matrix. These aberrant concentric membranous structures were similar to those observed in Atg5- 
Table 1 Primers used in this study

\begin{tabular}{lll}
\hline Gene & $5^{\prime}$-primer & \\
\hline Gapdh & CAAGGTCATCCATGACAACTTTG & GTCCACCACCCTGTTGCTGTAG \\
Evala & AGACAACCTTTCCTCCCAC & AGAGACAAAGTACAGAGCGGC \\
Anp & GTACAGTGCGGTGTCCAACA & TCTCCTCCAGGTGGTCTAGCA \\
Bnp & CACCGCTGGGAGGTCACT & GTGAGGCCTTGGTCCTTCAA \\
B-Mhc & GCATTCTCCTGCTGTTTCCTT & TGGATTCTCAAACGTGTCTAGTGA \\
Fibronectin & TGACCTGGAGGAAAACATTAAGA & AGCCCTGTATGTCTTCACACTG \\
Collagen I & CACGGAGGCCACCATTACT & CTTCAGGGCAATGACGTAGAT \\
Collagen III & CTCCTGGCAAGAATGGAGAT & AATCCACGAGCACCCTGA \\
\hline
\end{tabular}

deficient hearts. ${ }^{9}$ Finally, as the heart is rich in mitochondria, which are important organelles in energy metabolism, we investigated whether deficiency of Eva1a affected ATP generation. The ATP content was significantly lower in Eva1adeficient mice than in the other mice, indicating that normal mitochondrial function and mitochondrial energy metabolism may be affected in Eva1a-deficient mice by decreased autophagy, which may be one of the important reasons for the accelerated cardiac remodelling.

In summary, the present study demonstrates that Eva1a protects against cardiac remodelling and heart failure. The protective effects of Eva1a appear to involve autophagy via inhibition of the Mtor signalling pathway. Future studies should be conducted to explore the potential for Eva1a as a therapeutic target in heart failure.

\section{Materials and Methods}

Generation of tissue-specific Eva1a-deficient mice. Eva1 $a^{\text {floxflox }}$ mice with a C57BL/6 background were constructed by the Chinese Academy of Medical Sciences and bred at the Experimental Animal Center, Peking University Health Sciences Center (Beijing, China). The animal experimental protocol was approved by the Biomedical Research Ethics Committee of Peking University (LA 2010-048) and strictly adhered to the American Physiological Society's Guiding Principles in the Care and Use of Vertebrate Animals in Research and Training. Eight-week-old male C57BL/6 mice were provided by the Animal Department of Peking University Health Science Center (Beijing, China). The mice were housed in groups of four in a room with controlled temperature $\left(25 \pm 2^{\circ} \mathrm{C}\right)$, with free access to food and water. All the animal experiments were approved by Biomedical Research Ethics Committee of Peking University.

Mice harbouring a homozygous conditional null mutation in Eva1a floxfllox were crossed with transgenic $\alpha-M H C$ MerCreMer mice, which expressed a tamoxifeninducible Cre recombinase (MerCreMer) under the transcriptional control of the cardiomyocyte-specific $\alpha-M H C$ promoter, to generate conditional cardiomyocytespecific Eva1a knockout mice. All the mice had a C57BL/6 background, and Eva1atloxflox littermates without MerCreMer were used as the controls for this study. To selectively delete Eva1a in cardiomyocytes, we treated 8-week-old Eva1 filoxflox: $\alpha-M H C$

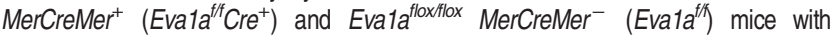
$80 \mathrm{mg} / \mathrm{kg}$ tamoxifen once a day for 3 days and analysed the mice. This dose resulted in the most rapid and effective knockdown of the Eva1a gene without any cardiotoxicity.

RNA isolation and real-time RT-PCR. Total RNA was prepared from mice tissues using TRIZOL reagent (15596, Invitrogen, Carlsbad, CA, USA), and cDNA was synthesized using Revert Aid First Strand cDNA Synthesis Kit (K1622, Thermo Scientific, Waltham, MA, USA). The expression of mRNA was analysed by quantitative real-time RT-PCR (Applied Biosystems, Waltham, MA, USA StepOne Plus) and normalized to the expression of the Gapdh housekeeping gene. Real-time RT-PCR was performed in triplicate for each sample. Table 1 presents a list of the primers used for real-time PCR.

Southern blot analysis. Genomic DNA from heart tissue was separated and purified by DNeasy Tissue kit (69504, Qiagen, Hilden, Germany). Southern blot analysis was performed according to the standard procedure. The isolated DNA was digested by EcoRV enzyme. The labelled probe was designed at the third exon using the forward primer $5^{\prime}$-TCTAAAGGACTCCGTGAA-3' and the reverse primer 5'-ACCTCTGGCTTCCATTCT-3'.

Western blot analysis. Briefly, the mice organs were collected and disrupted in lysis buffer containing protease inhibitors (Roche Diagnostics, Berlin, Germany). After centrifugation, the supernatant was collected, and equivalent amounts of protein were subjected to sodium dodecyl sulphate-polyacrylamide gel electrophoresis (SDS-PAGE) and transferred to a nitrocellulose membrane. The protein bands were visualized using DyLight 800/DyLight 680-conjugated secondary antibodies, and an infrared fluorescence image was obtained using an Odyssey infrared imaging system (LI-COR Biosciences, Lincoln, NE, USA). Western blot analyses were performed by ImageJ with anti-Gapdh (KM9002, Sungene, Tianjin, China), anti-Lc3b (SAB4200361, Sigma, St Louis, MO, USA), anti-Sqstm1 (PM045, MBL International, Japan), and anti-Eva1a (NB110-74787, Novusbio, Littleton, CO, USA) antibodies. Antibodies against Ulk1, Akt, Mtor, Erk1/2, Lkb1, Ampk, Rps6kb1, and Eif4ebp1 and against phosphorylated Ulk1, Akt, Mtor, Erk1/2, Lkb1, Ampk, Rps6kb1, and Eif4ebp1 were purchased from Cell Signaling Technology (Boston, MA, USA). Antibodies against Drp1, Tomm20, Pink1, Parkin, Bnip3, Mitofusin2, and Pgc1 were purchased from Abcam (Cambridge, UK). DyLight 800/DyLight 680conjugated secondary antibodies against mouse or rabbit lgG were purchased from Rockland Immunochemicals (Limerick, PA, USA).

Transmission electron microscopy. Cardiac tissue was initially fixed in $0.1 \mathrm{M}$ sodium phosphate buffer containing $3 \%$ glutaraldehyde $(\mathrm{pH} 7.4)$ and then fixed in $0.1 \mathrm{M}$ sodium phosphate buffer containing $1 \% \mathrm{OsO}_{4}(\mathrm{pH} 7.2)$ for $2 \mathrm{~h}$ at $4{ }^{\circ} \mathrm{C}$. The tissue was dehydrated in a graded ethanol series. Then the tissue was embedded in Ultracut (Leica Ultracut) and sliced into $60 \mathrm{~nm}$ sections. The ultrathin sections were stained with uranyl acetate and lead citrate, and observed under a JEM-1230 transmission electron microscope.

Echocardiographic analysis. Mice were anaesthetized with $1 \%$ isoflurane (Baxter Healthcare Corporation, New Providence, NJ, USA). Echocardiographic images were obtained using a Visualsonics high-resolution Vevo770 system (VisualSonics Inc., Toronto, ON, Canada). Two-dimensional parasternal long-axis and short-axis views were obtained at the level of the papillary muscle. Diastolic left ventricular posterior wall thickness (LVPW-d) and systolic left ventricular posterior wall thickness (LVPW-s) were measured to calculate the EF and FS. All the measurements were averaged from three consecutive cardiac cycles.

Quantitative histological analyses. After the mice were killed, the hearts were harvested and perfused in retrograde with cold phosphate-buffered saline (PBS), fixed with $4 \%$ paraformaldehyde for $8 \mathrm{~h}$, dehydrated in $20 \%$ sucrose for $24 \mathrm{~h}$, and embedded in paraffin. Serial $5 \mu \mathrm{m}$ thick sections were stained with H\&E for morphological analysis, or picrosirius red for the detection of fibrosis. For morphometric analysis, photographs of left ventricular sections cut from the same location of each heart were observed under $\times 400$ magnification (Leica Microsystems Imaging Solutions Ltd., Cambridge, UK). Interstitial fibrosis was visualized with picrosirius red staining, and the cardiac fibrosis volume fraction was calculated as the ratio of the stained fibrotic area to the total myocardial area. For TUNEL staining, heart tissues were embedded in a freezing matrix. Serial $7 \mathrm{~mm}$ cryostat sections were prepared and stored at $-20{ }^{\circ} \mathrm{C}$ until use. TUNEL assays 
were performed with the in situ cell death detection kit (Roche Applied Science, Indianapolis, IN, USA), according to the manufacturer's instructions. The sections were counterstained with 4', 6-diamidino-2-phenylindole (DAPI).

Detection of ATP levels. The ATP levels of heart tissue from mice were measured using a firefly luciferase-based ATP assay kit (Beyotime, Shanghai, China), according to the manufacturer's instructions. After the indicated treatments, cardiomyocytes were lysed and centrifuged at $12000 \times g$ for 5 min. Supernatants $(100 \mu \mathrm{l})$ were mixed with $100 \mu \mathrm{l}$ of ATP detection working dilution in a white 96-wel plate. Standard curves were also generated, and the protein concentration of each treatment group was determined using the Bradford protein assay. The total ATP levels were expressed as nmol/mg protein. This experiment was repeated three times.

Statistical analysis. The data are summarized as means \pm S.E.M. Differences between groups were compared using Prism 5 (GraphPad Software Incorporate, La Jolla, CA, USA) with unpaired two-tailed Student's t-test. A $P$-value $<0.05$ was considered statistically significant.

\section{Conflict of Interest}

The authors declare no conflict of interest.

Acknowledgements. This work was supported by the National Natura Science Foundation of China (31571052, 81570235, 81272432 and 81641018), the Beijing Natural Science Foundation (5162014), and the National Key Basic Research Program of China (2011CB910103 and 2015CB964501).

1. Levy D, Garrison RJ, Savage DD, Kannel WB, Castelli WP. Prognostic implications of echocardiographically determined left ventricular mass in the Framingham Heart Study. N Engl J Med 1990; 322: 1561-1566.

2. Frey N, Olson EN. Cardiac hypertrophy: the good, the bad, and the ugly. Annu Rev Physiol 2003; 65: 45-79.

3. Oparil S. Pathogenesis of ventricular hypertrophy. J Am Coll Cardiol 1985; 5: 57B-65B.

4. Minnaard-Huiban M, Emmen JM, Roumen L, Beugels IP, Cohuet GM, van Essen $\mathrm{H}$ et al. Fadrozole reverses cardiac fibrosis in spontaneously hypertensive heart failure rats: discordant enantioselectivity versus reduction of plasma aldosterone. Endocrinology 2008; 149: 28-31.

5. Tao H, Shi KH, Yang JJ, Huang C, Liu LP, Li J. Epigenetic regulation of cardiac fibrosis. Cell Signal 2013; 25: 1932-1938

6. Ma F, Li Y, Jia L, Han Y, Cheng J, Li H et al. Macrophage-stimulated cardiac fibroblas production of IL-6 is essential for TGF beta/Smad activation and cardiac fibrosis induced by angiotensin II. PLOS ONE 2012; 7: e35144.

7. Koshman YE, Patel N, Chu M, lyengar R, Kim T, Ersahin C et al. Regulation of connective tissue growth factor gene expression and fibrosis in human heart failure. J Card Fail 2013; 19: 283-294.

8. Sirish P, Li N, Liu JY, Lee KS, Hwang SH, Qiu H et al. Unique mechanistic insights into the beneficial effects of soluble epoxide hydrolase inhibitors in the prevention of cardiac fibrosis. Proc Natl Acad Sci USA 2013; 110: 5618-5623.

9. Nakai A, Yamaguchi O, Takeda T, Higuchi Y, Hikoso S, Taniike M et al. The role of autophagy in cardiomyocytes in the basal state and in response to hemodynamic stress. Nat Med 2007; 13: 619-624.

10. Kostin S, Pool L, Elsässer A, Hein S, Drexler HC, Arnon E et al. Myocytes die by multiple mechanisms in failing human hearts. Circ Res 2003; 92: 715-724.

11. Wang ZV, Rothermel BA, Hill JA. Autophagy in hypertensive heart disease. J Biol Chem 2010; 285: 8509-8514.

12. Gustafsson AB, Gottlieb RA. Autophagy in ischemic heart disease. Circ Res 2009; 104 $150-158$

13. De Meyer GR, Martinet W. Autophagy in the cardiovascular system. Biochim Biophys Acta 2009; 1793: 1485-1495
14. Mellor KM, Reichelt ME, Delbridge LM. Autophagy anomalies in the diabetic myocardium. Autophagy 2011; 7: 1263-1267.

15. Thomas RL, Roberts DJ, Kubli DA, Lee Y, Quinsay MN, Owens JB et al. Loss of MCL-1 leads to impaired autophagy and rapid development of heart failure. Genes Dev 2013; 27 : 1365-1377.

16. Sun M, Ouzounian M, de Couto G, Chen M, Yan R, Fukuoka $M$ et al. Cathepsin-L ameliorates cardiac hypertrophy through activation of the autophagy-lysosomal dependent protein processing pathways. J Am Heart Assoc 2013; 2: e000191.

17. Hu J, Li G, Qu L, Li N, Liu W, Xia D et al. TMEM166/EVA1A interacts with ATG16L1 and induces autophagosome formation and cell death. Cell Death Dis 2016; 7: e2323.

18. Wang L, Yu C, Lu Y, He P, Guo J, Zhang $C$ et al. TMEM166, a novel transmembrane protein, regulates cell autophagy and apoptosis. Apoptosis 2007; 12: 1489-1502.

19. Xu D, Yang F, He H, Hu J, Lv X, Ma D et al. Expression of TMEM166 protein in human normal and tumor tissues. Appl Immunohistochem Mol Morphol 2013; 21: 543-552.

20. Chang Y, Li Y, Hu J, Guo J, Xu D, Xie H et al. Adenovirus vector-mediated expression of TMEM166 inhibits human cancer cell growth by autophagy and apoptosis in vitro and in vivo. Cancer Lett 2013; 328: 126-134.

21. Li M, Lu G, Hu J, Shen X, Ju J, Gao Y et al. EVA1A/TMEM166 regulates embryonic neurogenesis by autophagy. Stem Cell Rep 2016; 6: 1-15.

22. Wight TN, Potter-Perigo S. The extracellular matrix: an active or passive player in fibrosis? Am J Physiol Gastrointest Liver Physiol 2011; 301: G950-G955.

23. Zhang S, Li G, Fu X, Qi Y, Li M, Lu G et al. PDCD5 protects against cardiac remodeling by regulating autophagy and apoptosis. Biochem Biophys Res Commun 2015; 461: 321-328.

24. Kageyama Y, Hoshijima M, Seo K, Bedja D, Sysa-Shah P, Andrabi SA et al. Parkinindependent mitophagy requires Drp1 and maintains the integrity of mammalian heart and brain. EMBO J 2014; 33: 2798-2813.

25. Grey JY, Connor MK, Gordon JW, Yano M, Mori M, Hood DA. Tom20-mediated mitochondrial protein import in muscle cells during differentiation. Am J Physiol Cell Physiol 2000; 279: C1393-C1400

26. Tian W, Li W, Chen Y, Yan Z, Huang X, Zhuang H et al. Phosphorylation of ULK1 by AMPK regulates translocation of ULK1 to mitochondria and mitophagy. FEBS Lett 2015: 589.

27. Mizushima N, Levine B, Cuervo AM, Klionsky DJ. Autophagy fights disease through cellular self-digestion. Nature 2008; 451: 1069-1075.

28. Cecconi F, Levine B. The role of autophagy in mammalian development: cell makeover rather than cell death. Dev Cell 2008; 15: 344-357.

29. Loos B, Lochner A, Engelbrecht AM. Autophagy in heart disease: a strong hypothesis for an untouched metabolic reserve. Med Hypotheses 2011; 77: 52-57.

30. Fitzwalter BE, Thorburn A. Recent insights into cell death and autophagy. FEBS J 2015; 282: 4279-4288.

31. Li L, Khatibi NH, Hu Q, Yan J, Chen C, Han J et al. Transmembrane protein 166 regulates autophagic and apoptotic activities following focal cerebral ischemic injury in rats. Exp Neurol 2012; 234: 181-190.

32. Varga ZV, Giricz Z, Liaudet L, Haskó G, Ferdinandy P, Pacher P. Interplay of oxidative, nitrosative/nitrative stress, inflammation, cell death and autophagy in diabetic cardiomyopathy. Biochim Biophys Acta 2015; 1852: 232-242.

33. Levine B, Mizushima N, Virgin HW. Autophagy in immunity and inflammation. Nature 2011; 469: 323-335.

(c) (i) Cell Death and Disease is an open-access journal published by Nature Publishing Group. This work is licensed under a Creative Commons Attribution 4.0 International License. The images or other third party material in this article are included in the article's Creative Commons license, unless indicated otherwise in the credit line; if the material is not included under the Creative Commons license, users will need to obtain permission from the license holder to reproduce the material. To view a copy of this license, visit http://creativecommons.org/licenses/by/4.0/

(C) The Author(s) 2017

Supplementary Information accompanies this paper on Cell Death and Disease website (http://www.nature.com/cddis) 